\title{
Intracardiac Pneumonectomy
}

National Cancer Institute

\section{Source}

National Cancer Institute. Intracardiac Pneumonectomy. NCI Thesaurus. Code C120275.

Excision of a lung and part of the pericardium. 